\title{
The Single-Cell Pathology Landscape of Breast Cancer
}

Hartland W. Jackson ${ }^{1, *}$, Jana R. Fischer ${ }^{1,2, \star}$, Vito R.T. Zanotelli ${ }^{1,2}$, H. Raza Ali ${ }^{1,3}$, Robert Mechera $^{4}$, Savas D. Soysal ${ }^{5,6}$, Holger Moch ${ }^{7}$, Simone Muenst ${ }^{8}$, Zsuzsanna Varga ${ }^{7}$, Walter P. Weber $^{4}$, and Bernd Bodenmiller ${ }^{1}$

${ }^{1}$ Department of Quantitative Biomedicine, University of Zurich, Switzerland

${ }^{2}$ Life Science Zurich Graduate School, ETH Zurich and University of Zurich, Switzerland ${ }^{3}$ CRUK Cambridge Institute, University of Cambridge, Cambridge, UK

${ }^{4}$ Department of Surgery, University of Basel and University Hospital Basel, Switzerland

${ }^{5}$ Visceral Surgery Research Laboratory, Clarunis, Department of Biomedicine, University of

Basel, Basel, Switzerland

${ }^{6}$ Department of Surgery, Clarunis University Center for Gastrointestinal and Liver Diseases

Basel, Basel, Switzerland

${ }^{7}$ Institute of Pathology and Molecular Pathology, University Hospital Zurich, Zurich, Switzerland.

${ }^{8}$ Institute of Pathology and Genetics, University Hospital Basel, Switzerland

*Authors contributed equally to this work.

Correspondence should be addressed to

B.B. (bernd.bodenmiller@uzh.ch) 


\section{ABSTRACT}

Single-cell analyses have revealed extensive intra- and inter-patient cancer heterogeneity ${ }^{1}$, but complex single-cell phenotypes and their spatial context are not yet reflected in the histologic stratification that is the foundation of many clinical decisions. Here, we used imaging mass cytometry ${ }^{2}$ to simultaneously quantify 35 biomarkers resulting in 720 highdimension immunohistochemistry pathology images of tumor tissue from 352 breast cancer patients for whom long-term survival data were available. Spatial, single-cell analysis identified tumor and stromal single-cell phenotypes, their organization and heterogeneity, and enabled categorization of breast cancer cellular architecture based on cellular composition and tissue organization. Our analysis revealed multi-cellular features of the tumor microenvironment and novel breast cancer subgroups associated with distinct clinical outcomes. Thus, spatially resolved, single-cell analysis can characterize intra-tumor phenotypic heterogeneity in a disease-relevant manner with the potential to inform patient-specific diagnosis.

\section{Main}

Histologic and phenotypic differences between tumors guide cancer diagnosis, prognosis, and selection of treatment. Currently, breast cancer patients are graded based on tumor structure and cellular morphology, and subcategorized when more than $1 \%$ of tumor cells contain hormone receptors or more than $10 \%$ express high levels of HER2 or have amplified HER2 ${ }^{3-5}$. This leaves a large portion of cells uncharacterized even though additional molecular subclasses and morphologic features have been identified as prognostic ${ }^{6-9}$. It is clear that clonal evolution and spatially distinct tumor microenvironments drive inter- and intra-patient cellular 
heterogeneity and hinder effective treatment ${ }^{10-14}$. Using highly multiplexed imaging ${ }^{2,15,16}$, multiple complex cellular phenotypes have been identified within the context of the tumor microenvironment, enabling refined histopathology classification of clinical tissue samples ${ }^{17-20}$. Here, harnessing topographical single-cell network analysis of high-dimensional mass cytometry images, we quantified spatial inter- and intra-tumor single-cell heterogeneity and identified spatially resolved features and novel breast cancer subtypes that are associated with clinical outcome.

\section{Spatially resolved single-cell phenotypes}

To comprehensively quantify the cellular heterogeneity and spatial organization of breast cancer tissue, we designed a breast-histology-specific imaging mass cytometry (IMC) panel (Extended Data 1) to image samples from 281 tumors representing all clinical subtypes and pathology grades (Supplementary Table 1). IMC combines immunohistochemistry staining using metal isotope-labelled antibodies with laser ablation and mass spectrometry-based detection to produce high-dimension images (Figure 1a)². Our 35-antibody panel simultaneously quantified clinically established breast cancer targets estrogen receptor (ER), progesterone receptor (PR), and HER2, proliferation marker Ki-67, markers of epithelial, mesenchymal, immune, and endothelial lineages, and targets that provide insight into signaling pathways, oncogenes, and epigenetics (Extended Data 1, Supplementary Table 2). IMC produces images comparable to immunofluorescence or immunohistochemistry but with capacity for highly multiplexed staining $^{2,21}$ (Extended Data 2 and 3). Images were segmented into single cells and tumor and stromal regions using a random forest pixel classifier (Ilastik), and CellProfiler ${ }^{17,20}$. We identified 
855,668 cells in 381 images (289 tumor, 87 healthy breast and 5 liver controls), and quantified marker expression and spatial features of each cell (Figure 1a). Clustering with PhenoGraph ${ }^{22}$ identified cell phenotype clusters, hereafter referred to as phenotypes, of endothelial, T and B cell, macrophage, and stromal cell populations as well as 59 diverse tumor cell phenotypes. Similar to Wagner et $a .^{1}{ }^{1}$, some tumor phenotypes were unique to individual patients (Figure 1b, c, Extended Data 4a). To identify common cellular subtypes within this diversity, we defined 14 tumor-cell metaclusters by hierarchical clustering of the PhenoGraph-defined tumor single-cell phenotypes (Figure 1c, Extended Data 5a).

Tumors from every clinical subtype contained fibroblast, endothelial, and immune cell populations at similar densities but were enriched in tumor cell populations with variable expression of cytokeratins, hormone receptors, and HER2 reflective of clinical subtype (Figure 1c, Extended Data 6a). Across all patients, immune cells were excluded from the cohesive tumor mass, although immune and fibroblast cells did infrequently infiltrate the tumor mass and rare $\mathrm{HR}^{\text {low/- }}$ cells lacking cytokeratins invaded past the tumor-stroma front in some samples (Figure 1c, Extended Data 4). Tumor regions contained various luminal $\mathrm{HR}^{+}$epithelial cell phenotypes identified by combinations of ER, PR, GATA3, E-cadherin, and multiple cytokeratins, but hormone receptors were also expressed without cytokeratins in a few cases (metacluster 26) (Figure 1c, Extended Data 6b). Of the luminal cytokeratins (CK7, CK8/18, and CK19), only CK7 was associated with specific luminal tumor cell subsets (metaclusters 19, 20) (Figure 1c). HER2 expression was not a defining metacluster feature but was observed at different levels in multiple phenotypes. Phenotypes without hormone and HER2 receptor expression (characteristics of triple-negative breast cancer (TNBC)) included metaclusters with high levels of Ki-67, p53, EGFR, and hypoxia 
marker CAIX (metaclusters 15-17), basal cytokeratins (metacluster 18), and even luminal cytokeratins (PG clusters within metaclusters 19, 22) (Figure 1c).

\section{Multi-cellular breast cancer architecture}

Based on these single-cell phenotypes, we sought to define patterns of multi-cellular architecture in breast tumor tissue (Figure 2a). We evaluated regional correlations between cellular metaclusters to determine whether cells co-occurred across all images, and used permutation test-based neighborhood analysis ${ }^{17}$ to quantify cell co-localization and identify statistically significant interaction or avoidance between pairs of cell phenotypes (Figure $2 b$ ). Tumor cell phenotypes were rarely correlated: Each individual tumor contained many homotypic interactions between similar cells and few heterotypic tumor cell interactions (Figure 2b, box \#1). Generally, heterotypic interactions were associated with regionally unique structures such as blood vessels (Figure $2 \mathrm{~b}$, box \#2) or with distinct epithelial (box \#1) or stromal cell-dense areas where immune cells interacted (box \#3). Uni-directional interactions were also observed where supporting fibroblasts enclosed endothelial cells in large blood vessels and where stromal cells surrounded tumor cells at the tumor-stroma interface (Figure $2 \mathrm{~b}$, boxes \#2 and \#4). T cells and proliferating epithelial cells were observed in the vicinity of endothelial cells, and their proportions were correlated across images; T cells were surrounded by endothelial cells (e.g., inside vessels), not the reverse (Figure $2 \mathrm{~b}$, boxes \#5 and \#6). The neighborhood analysis revealed interaction signatures that distinguished well-separated or stromal-interactive tumor architectures that were related to pathologist-scored tumor grade (Extended Data 7) ${ }^{17}$. 
Tissue function is implemented by multicellular units, which we call communities, that consist of higher order, rather than paired, interactions between one or more cell phenotypes. We identified communities by first constructing a topological neighboring cell interaction network and then applying a graph-based community detection approach using the Louvain algorithm (Figure 2a) ${ }^{23}$. Applied only to tumor cells, community detection identified dense epithelial patches of different sizes, termed tumor communities (TCs); when applied to all cells microenvironment communities (MCs) containing tumor and stromal cell components were identified (Figure 2a). Using PhenoGraph, we grouped multi-cell communities according to community size and tumor cell phenotypes (TCs) or all cells, but agnostic to tumor cell type (MCs) . Tumor communities were mostly dominated by a single cellular metacluster and were separated based on absolute number of cells (Figure 2c, d, TCs 4, 7, 18; Supplementary Images). Some microenvironment communities consisted of fibroblasts that interacted with a variety of tumor cells (MCs 2, 5, 8); others showed sparse stroma content (MCs 14, 17, 18, 20, 21, 22) or were enriched for T cells (MCs 19, 25, 30), macrophages (MC 27), large networks of T and B cells (MC 1), or endothelial cells (MCs 13, 6, 30, 25, 7) (Figure 2e, f). Fibroblast-enriched communities had few interacting immune cells, aligning with the known roles of fibroblasts as agents of tumor desmoplasia and immune exclusion ${ }^{24}$.

\section{Single-cell pathology subgroups are related to clinical outcome}

We next investigated how the organization of single cells into communities contributes to breast cancer tissue architecture and tumor subtypes ${ }^{3}$. Cells from multiple cellular metaclusters were found in every clinically defined breast cancer subtype (Extended Data 6a), 
supporting the conclusion that general pathology classification does not fully elucidate inter-and intra-patient cellular heterogeneity ${ }^{6,8}$. We reasoned that the single-cell pathology landscape would provide a higher resolution patient classification than classic histology-based clinical subtypes. Patient tumors were grouped based on the tumor cell metacluster composition using unsupervised clustering, identifying 18 single-cell pathology (SCP) subgroups that split the classic clinical subtypes (Figure 3a, Extended Data 8a, Supplementary Table 3). SCP subgroups had various proportions of the epithelial communities (Figure 3b), and individual SCP subgroups had distinct clinical outcomes when compared to all other patients, to SCP subgroups of the same clinical classification, and to other SCP subgroups containing similar cellular metaclusters but different architectures (Figure 4a-h, Supplementary Tables 4 and 5).

$\mathrm{HR}^{+}$clinically defined tumors were divided into those strongly enriched in cells with high expression of hormone receptors (SCPs 1-5 and 12) and tumors with few $\mathrm{HR}^{\mathrm{h} / /+}$ cells surrounded by many cells that expressed only low levels or lacked HRs (SCPs 6-10 and 11) that are currently not clinically classified (Figure 3a, Extended Data 6 and 8a, Supplementary Images). SCP 1, which contained predominantly metacluster $23\left(\mathrm{CK}^{+} / \mathrm{HR}^{\text {hi }}\right.$ tumor cells), was only associated with patients who did not succumb to disease. Conversely, SCP 3, which contains the same cellular metaclusters but differs in structure, with smaller communities and relatively higher proportions of $\mathrm{CK}^{\text {low }} / \mathrm{HR}^{\text {low }}$ metacluster 22 and 25 cells, was associated with poor prognosis, as were SCPs 6 and 9, which involve predominantly $\mathrm{CK}^{\text {low }} / \mathrm{HR}^{\text {low }}$ cells (Figure 3a, Figure 4c, e, Extended Data 9). SCP 2, containing $\mathrm{CK}^{+} / \mathrm{HR}^{+}$cells, was significantly enriched in the $\mathrm{HR}^{+} / \mathrm{HER}^{+}$clinical subtype, which was otherwise dominated by $\mathrm{CK}^{\text {low }} / \mathrm{HR}^{\text {low }}$ metacluster 22 (Figure 3a, Extended Data 6, and 8a). SCPs 11 and 12 were characterized by $C K 7^{+}$cells primarily from metaclusters 20 and 19 , 
respectively. SCP 11 overlapped with the clinically assigned $\mathrm{HR}^{-} / \mathrm{HER}^{+}$tumor type, and, although this clinical subtype usually has poor outcomes ${ }^{3,5}$, SCP 11 patients had significantly better outcomes than other patients in this cohort. In contrast, the small number of CK7 ${ }^{+}$SCP 12 patients, predominantly clinically assigned as $\mathrm{HR}^{+} / \mathrm{HER2} 2^{-}$, did not survive long term (Figure 3a, Figure 4a, c, e, Extended Data 6 and 8a). Tumors from patients with high-risk TNBC contained distinct cell types including cells with cytokeratin expression suggestive of a luminal, not myoepithelial, cell of origin (Figure 1c, Figure 3a, Extended Data 6 and 8a). TNBC phenotypes without luminal epithelial markers and with high levels of hypoxic, $\mathrm{p} 53^{+} / \mathrm{EGFR}^{+}$, basal, or proliferative markers distinguished SCPs 13, 14, 15, and 17 with poor outcome (Figures 1c, Figure 3a, Extended Data 6 and 8a). SCP 16 tumors were $p 53^{+}$and expressed apoptotic markers, and interestingly, patients with tumors of this group did not succumb to disease even though they were clinically classified as TNBC (Figure 4f, Extended Data 8a).

By mapping the cellular spatial organization of these tumors, we observed variable structures and cellular densities, and relationships between cellular phenotype and tissue organization (Figure 3a, b). Heterogeneous tumors consisted of multiple phenotypically pure communities indicated by many bands on the heatmap, whereas homogeneous tumors organized in one epithelial sheet or with similar communities of different sizes have only a few clustered bands (Figure $3 b$ ). Most tumors were dominated by a single tumor-cell metacluster and few community types, but tumors in SCP 8 and some in SCP 10 were unusually heterogeneous, consisting of multiple epithelial cellular metaclusters at similar proportions localized to spatially distinct communities (Figure 3b, Extended Data 6). Patients in SCP8 with these heterogeneous tumors had very poor outcomes (Figure 4d, e). Overall, intra-tumor phenotypic heterogeneity 
was spatially segregated into separate tumor communities as opposed to heterogeneous tumor masses, and patients with tumors with greater spatio-phenotypic heterogeneity had poorer outcomes.

Unlike tumor cell phenotypes, the identified stromal cell phenotypes were present in every clinical subtype at similar densities (Figure 1c, Extended Data 6). We therefore investigated whether the tumor-stromal microenvironment communities were more informative than mere stromal phenotype content. When we hierarchically clustered images by the presence of microenvironment communities, 11 groups, which we term stromal environments (SEs), were revealed; some were enriched in one microenvironment community (single column in Extended Data 7a), whereas others contained mixtures of communities (multiple columns). Neighbor analysis detected distinct cell-cell interactions within each SE (Extended Data 7b). Some stromal environments included large epithelial networks with sparse stroma (SEs 7 and 10, made of MCs 17 or 18), others involved vascularized regions (SE9, involving MCs 6 and/or 13), different fibroblast cell phenotypes such as vimentin ${ }^{\text {hi }}$ (SEs 4, 6, and 9, involving metacluster 8 in many

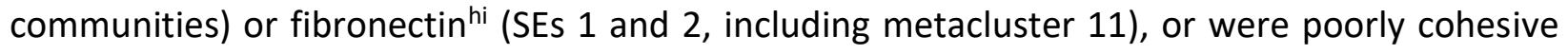
and made up of many small communities (SEs 2, 5, and 6).

We found that SEs were associated with SCP subgroups and specific tumor cell phenotypes. For example, hypoxic SCP 17 TNBCs were commonly classified as large, stromadeficient epithelial sheets (SEs 7), and TNBCs SCP 13-16 were associated with T cell-enriched (SES 5 and 8) or macrophage-enriched (SE 3) stromal environments (Extended Data 7 and 8b). $\mathrm{HR}^{+}$ tumors are likely to be immune-cold, but some contained rare and localized immune-enriched communities (SE 5); the $\mathrm{HR}^{+}$tumors never contained an exclusively immune-hot stromal 
environment (SE 8) like those observed in TNBCs and the highly heterogeneous SCP 8 (Extended Data 7 and $8 \mathrm{~b})$. $\mathrm{HR}^{+}$tumors were associated with a range of fibroblast-enriched stromal environments with small elongated fibroblasts interacting with $\mathrm{CK}^{+} / \mathrm{HR}^{\text {low }}$ epithelial cells in SEs 1, 2, and 5 and vimentin ${ }^{\text {hi }}$ stroma associated with $H R^{\text {hi }}$ tumor cells in SEs 4 and 6 (Figure 2b, c, Extended Data 8b). Stromal environments were related to tumor phenotype, but only fragmented SE 6 containing proliferative vimentin ${ }^{\text {hi }}$ fibroblasts was independently associated with poorer disease-free and overall survival (Figure 4h, Extended Data 8b and 9, Supplementary Tables 6 and 7).

Compared to clinically defined subtypes, SCP grouping improved the ability to predict a patient's overall survival using Cox proportional hazards modeling (Supplementary Table 8). In order to identify features associated with patient risk not captured by clinical grading and classification, we investigated the epithelial and stromal single-cell and community contributions to the model. Almost no single-cell phenotypes or cellular metaclusters were independently associated with outcome (not shown); however, spatially defined cell communities were (Figure 4i). For certain cell types, large tumor cell communities were related to better outcome, whereas similar networks of small size were related to poor outcome (Figure 4i, TCs 12 vs. 13,17 vs. 23, 5 vs. 15). In addition, the microenvironment community MC 6, characterized by vascularization with T cell involvement, was significantly associated with increased risk of death even though it was more commonly found in the low-risk $\mathrm{HR}^{+}$clinical subgroups than other subgroups (Figure 4i, Extended Data 7). In contrast, highly T cell infiltrated MC 19 and macrophage-enriched MC 27 were significantly associated with better patient outcomes even though inflammation is more common in high-risk TNBC tumors than other clinical subgroups (Figure 4i, Extended Data 7). 
Thus, SCP-defined tumor types and tumor and stromal architectures could inform prognosis beyond current clinical classifications.

\section{Quantification of intra-tumor heterogeneity}

We investigated reproducibility and spatial variability of SCP classifications in two central and two peripherally located tumor regions from 72 patients in an independent cohort resulting in 344 additional images containing 411,410 cells (Supplementary Table 9). We used the same analytical approach described above to independently define single-cell phenotypes, match them to cellular metaclusters and to classify each imaged region into SCP subgroups and stromal architectures (Extended Data 5b and 10a). All cellular metaclusters and SCP subgroups identified in the first cohort were present in the second cohort. Likely due to the patient selection strategy, which enriched the second cohort in metastatic low-grade tumors, we observed a higher proportion of subgroups involving $\mathrm{CK}^{\text {low }} / \mathrm{HR}^{\text {low }}$ single-cell phenotypes such as SCP 3,6 , and 9 in this cohort (Extended Data 10a). For each region of each tumor, we quantified the spatial variability of cell phenotypes using Shannon entropy, and the difference in cellular content relative to the patient's overall single-cell phenotype distribution (all regions) was determined using Kullback-Leibler divergence. Most patients had only moderate inter-region heterogeneity (Extended Data 10b). Distinctly divergent regions were more commonly identified as SCP 2, which consists of $\mathrm{CK}^{+} / \mathrm{HR}^{+}$cells, or SCP 7, containing Epithelial low cells (Extended Data 10b, c). Approximately $40 \%$ of tumors had identical classification in all regions, and $60 \%$ had one or more regions that did not agree with the whole tumor classification (Extended Data 10d). However, in 
the majority of cases SCP classification of individual regions matched the tumor-wide classification.

SCP subgroups had varying levels of regional heterogeneity. SCP subgroups found in TNBCs were phenotypically homogenous (the exception being basal $\mathrm{CK}^{+}$SCP 13). SCP subgroups associated with $\mathrm{HR}^{+}$patients with relatively poor prognosis, such as $\mathrm{CK}^{\text {low }} / \mathrm{HR}^{\text {low }} \mathrm{SCPs} 6,7,9,10$, had substantial spatial heterogeneity (Extended Data 10e). Different SCP subgroups containing similar cell types occasionally co-occurred in the same tumor, but SCP 9 was always accompanied by SCP 6 regions, and the two were associated with similar outcomes (Figure 4e, Extended Data 10e). Spatially heterogeneous $\mathrm{HR}^{\text {low }}$ and $\mathrm{CK}^{\text {low }} \mathrm{SCP}$ subgroups 6-10 have indistinguishable outcomes from the population average, and based on their regional heterogeneity it is statistically likely that unsampled phenotypes influenced classification accuracy (Extended Data 10e). We observed that heterogeneous regions classified as SCP 8 or 10 were always accompanied by multiple additional tumor subtypes (Extended Data 10e). These may be multiclonal or highly plastic tumors. Subsampling was sufficient to identify and stratify homogenous low-risk HR hi and high-risk TNBCs, but increased sampling may be needed to accurately identify $H^{\text {low }}$ tumor phenotypes and tumors with considerable intra-tumor heterogeneity.

\section{Discussion}

This systematic, multidimensional interrogation of breast cancer histology generated a detailed spatial map of single-cell phenotypes and cellular communities related to disease. We demonstrated that single-cell pathology can better segregate patients with distinct clinical

outcomes than the current clinical subtyping strategy. Analysis of multi-cellular structures 
revealed that phenotypic heterogeneity in tumors was spatially localized to distinct regions or lesions. Moreover, the multi-cellular structures yielded patient outcome-relevant information superior to single-cell data alone. We identified co-occurring breast cancer phenotypes and observed that phenotypic and spatial heterogeneity varied between clinically established subtypes. This work suggests that multi-cellular spatial information is medically relevant and provides a basis for future study of how spatial and phenotypic tissue features influence disease progression.

\section{References}

Bibliography

1. Wagner, J. et al. A Single-Cell Atlas of the Tumor and Immune Ecosystem of Human Breast Cancer. Cell 177, 1330-1345.e18 (2019).

2. Giesen, C. et al. Highly multiplexed imaging of tumor tissues with subcellular resolution by mass cytometry. Nat. Methods 11, 417-422 (2014).

3. Coates, A. S. et al. Tailoring therapies--improving the management of early breast cancer: St Gallen International Expert Consensus on the Primary Therapy of Early Breast Cancer 2015. Ann. Oncol. 26, 1533-1546 (2015).

4. Hammond, M. E. H. et al. American Society of Clinical Oncology/College Of American Pathologists guideline recommendations for immunohistochemical testing of estrogen and progesterone receptors in breast cancer. J. Clin. Oncol. 28, 2784-2795 (2010).

5. Wolff, A. C. et al. Human epidermal growth factor receptor 2 testing in breast cancer: 
american society of clinical oncology/college of american pathologists clinical practice guideline focused update. J. Clin. Oncol. 36, 2105-2122 (2018).

6. Curtis, C. et al. The genomic and transcriptomic architecture of 2,000 breast tumours reveals novel subgroups. Nature 486, 346-352 (2012).

7. Sørlie, T. et al. Gene expression patterns of breast carcinomas distinguish tumor subclasses with clinical implications. Proc Natl Acad Sci USA 98, 10869-10874 (2001).

8. Cancer Genome Atlas Network. Comprehensive molecular portraits of human breast tumours. Nature 490, 61-70 (2012).

9. Beck, A. H. et al. Systematic analysis of breast cancer morphology uncovers stromal features associated with survival. Sci. Transl. Med. 3, 108ra113 (2011).

10. Bedard, P. L., Hansen, A. R., Ratain, M. J. \& Siu, L. L. Tumour heterogeneity in the clinic. Nature 501, 355-364 (2013).

11. Dagogo-Jack, I. \& Shaw, A. T. Tumour heterogeneity and resistance to cancer therapies. Nat. Rev. Clin. Oncol. 15, 81-94 (2018).

12. McGranahan, N. \& Swanton, C. Biological and therapeutic impact of intratumor heterogeneity in cancer evolution. Cancer Cell 27, 15-26 (2015).

13. Focke, C. M., Decker, T. \& van Diest, P. J. Intratumoral heterogeneity of Ki67 expression in early breast cancers exceeds variability between individual tumours. Histopathology 69, 849-861 (2016).

14. Rye, I. H. et al. Intratumor heterogeneity defines treatment-resistant HER2+ breast tumors. Mol. Oncol. 12, 1838-1855 (2018).

15. Angelo, M. et al. Multiplexed ion beam imaging of human breast tumors. Nat. Med. 20, 
436-442 (2014).

16. Gerdes, M. J. et al. Highly multiplexed single-cell analysis of formalin-fixed, paraffinembedded cancer tissue. Proc Natl Acad Sci USA 110, 11982-11987 (2013).

17. Schapiro, D. et al. histoCAT: analysis of cell phenotypes and interactions in multiplex image cytometry data. Nat. Methods 14, 873-876 (2017).

18. Keren, L. et al. A Structured Tumor-Immune Microenvironment in Triple Negative Breast Cancer Revealed by Multiplexed Ion Beam Imaging. Cell 174, 1373-1387.e19 (2018).

19. Carvajal-Hausdorf, D. E. et al. Multiplexed (18-Plex) Measurement of Signaling Targets and Cytotoxic T Cells in Trastuzumab-Treated Patients using Imaging Mass Cytometry. Clin. Cancer Res. 25, 3054-3062 (2019).

20. Damond, N. et al. A map of human type 1 diabetes progression by imaging mass cytometry. Cell Metab. 29, 755-768.e5 (2019).

21. Bodenmiller, B. Multiplexed Epitope-Based Tissue Imaging for Discovery and Healthcare Applications. Cell Syst. 2, 225-238 (2016).

22. Levine, J. H. et al. Data-Driven Phenotypic Dissection of AML Reveals Progenitor-like Cells that Correlate with Prognosis. Cell 162, 184-197 (2015).

23. Blondel, V. D., Guillaume, J.-L., Lambiotte, R. \& Lefebvre, E. Fast unfolding of communities in large networks. J. Stat. Mech. 2008, P10008 (2008).

24. Kalluri, R. The biology and function of fibroblasts in cancer. Nat. Rev. Cancer 16, 582-598 (2016). 


\section{Figure legends}

Figure 1: Single-cell phenotypes in high-dimension histopathology of breast cancer.

(a) Schematic of IMC acquisition of multiplexed images from 281 breast cancer patients and the analyses of single-cell phenotypes, metaclusters, stromal-cell organization and architecture, tumor and patient subclassification, and patient overall survival. (b) tSNE map of 171,288 subsampled single cells from high-dimension images of breast tumors colored by cell-type metacluster identifier. (c) Heatmap showing the z-scored mean marker expression or distance to tumor-stroma interface for each PhenoGraph cluster, colored according to metacluster identifier. The absolute cell counts of each PhenoGraph cluster are displayed as a bar plot. In the bubble plot, circle size shows the relative proportion of all cells in a clinical subtype that come from each cluster, and circle opacity shows the proportion of each cluster present in the different clinical subtypes.

Figure 2: A global map of the cellular neighborhoods and cell interaction networks of breast cancer.

(a) Representative image depicting the different steps in the spatial analysis. Images show (left to right) pseudo-colored IMC, single-cell mask of the same field of view labeled by cellular metacluster identifier, the topologic cell interaction network, modular regions of the tumor network identifying epithelial communities labeled in color, and modular regions in the tumorstroma network identified as tumor microenvironment communities. Scale bar $=100 \mu \mathrm{m}$. (b) Heatmap in which squares visualize Pearson correlation of cell phenotype proportions across all measured tissue regions ( $n=367$ images) and circles indicate significant pairwise cell type 
interaction or avoidance summarized across the two-sided permutation tests on the individual images ( $n=367$ images, 1000 permutations each). Circle color indicates percentage of images and size represents number of images with a significant cell-cell interaction or avoidance $(p<0.01)$. Highlighted interactions include 1) tumor epithelium , 2) endothelium, 3) immune, 4) surrounding stroma, 5) endothelium and T cells, and 6) proliferating epithelium surrounding endothelial cells. Non-symmetric highlight colors indicate examples of directional interaction. Uniquely colored (c-d) epithelial communities ( $n=8495)$ and (e-f) microenvironment communities ( $n=12,854$ ) clustered by PhenoGraph based on min-max normalized absolute numbers of cells from each cellular metacluster and visualized $(c, e)$ on a tSNE map and $(d, f)$ in stacked bar plots indicating the average number of cells from each cellular metacluster.

Figure 3: Single-cell pathology identifies subgroups of breast cancer patients.

(a) Hierarchically clustered stacked bar plot of cell-type metacluster densities in each tumor. Colored columns indicate clinical subtypes, and SCP subgroups. (b) Heatmap indicating proportions of different epithelial communities present within each image.

Figure 4: Single-cell pathology subgroups have distinct clinical outcomes.

Kaplan-Meier curves of overall survival for each patient group ( $n=278$ patients total) based on (a) clinical subtype, (b) clinical grade, (c-f) SCP subgroup, or (g-h) stromal environment. Two-sided logrank test 00.05 compared to all other samples, $\star \mathrm{p}<0.05$ compared to similar subgroups, p $<0.05$ compared to other $\mathrm{HR}^{+} / \mathrm{HER}^{-}$patients (for exact $\mathrm{p}$-values see SI Tables 4 and 6). (i) Relative hazard ratios and $95 \%$ confidence intervals of disease-specific overall survival for densities of tumor $(\mathrm{T})$ and microenvironment (ME) cellular communities and clinical categories 
(molecular subtype and grade) estimated by Cox proportional hazards model ( $n=266$ patients, $n=15$ patients only containing communities $<10$ cells were excluded).

\section{Methods}

\section{Clinical data}

The samples of tumors from the described patient cohorts were obtained from University Hospital Basel and University Hospital Zurich. The cohort from University Hospital Basel includes 281 patients who were not selected for any clinical or histologic features. Pathologists recorded the available patient metadata (Supplementary Table 1 ) and evaluated the suitability of tissue sections for tissue microarray (TMA) construction ${ }^{25}$. The TMA contains one $0.8-\mathrm{mm}$ tumor core per patient, in some cases an additional matched healthy breast tissue sample, and a few control samples (liver tissue). The cohort from University Hospital Zurich is comprised of 72 patients; the samples include four 0.6-mm cores from four different regions of each tumor as described in Kündig et al. ${ }^{26}$. Tumor cores were punched from two central and two peripheral areas that averaged $1 \mathrm{~cm}$ in distance between regions. Samples were selected to contain equal proportions of the different tumor grades as well as patients with and without lymph node metastases (Supplementary Table 9). In total 720 images were acquired that varied in size and localization in the tumor. This project was approved by the local Commission of Ethics (ref. no. 2014-397 and 2012-0553).

\section{Panel}


An antibody panel was designed to target epitopes specific for breast cancer as well as markers for cell cycle and phospho-signaling and to distinguish epithelial, endothelial, mesenchymal, and immune cell types (Extended Data 1, Supplementary Table 2). Clone information is available in Supplementary Table 2.

\section{Preparation and staining}

Tissue samples were formalin-fixed and paraffin-embedded at the University Hospitals of Basel and Zurich. The above described antibody panel was used to stain the tissue sections ${ }^{27}$. Tissue sections were dewaxed in xylene overnight and rehydrated in a graded series of alcohol (ethanol:deionized water 100:0, 90:10, 80:20, 70:30, 50:50, 0:100; 5 min each). In a $95^{\circ} \mathrm{C}$ water bath, heat-induced epitope retrieval was conducted in Tris-EDTA buffer at pH 9 for $20 \mathrm{~min}$. The tissue microarrays were immediately cooled and then blocked with $3 \%$ BSA, $5 \%$ goat serum in TBS for $1 \mathrm{~h}$. Samples were incubated overnight at $4{ }^{\circ} \mathrm{C}$ in primary antibody at $7.5 \mathrm{~g} / \mathrm{L}$ diluted in TBS/0.1\% Triton X-100/1\% BSA. Tissue samples were washed twice with TBS/0.1\% Triton X-100 and twice with TBS and dried before imaging mass cytometry measurements.

For combined immunofluorescence and imaging mass cytometry staining, tissues were stained overnight at $4{ }^{\circ} \mathrm{C}$ with primary metal-conjugated mouse HER2 $\left({ }^{151} \mathrm{Eu}\right)$ and rabbit panCytokeratin $\left({ }^{175} \mathrm{Lu}\right)$ antibodies prior to washing and the mixed addition of fluorescent and metalconjugated anti-mouse (AF488, $\left.{ }^{165} \mathrm{Ho}\right)$ and anti-rabbit (AF555, $\left.{ }^{159} \mathrm{~Tb}\right)$ secondary stains for $1 \mathrm{~h}$ at room temperature. A cover slip was added, and tissue was imaged for fluorescence signal. Subsequently, the cover slip was removed, and samples were washed, dried, and subjected to mass cytometry laser ablation and acquisition. 


\section{Imaging mass cytometry}

Images were acquired using a Hyperion Imaging System (Fluidigm). The largest square area from each core of a tissue microarray was laser ablated in a rastered pattern at $200 \mathrm{~Hz}$, and raw data preprocessing was completed using commercial acquisition software (Fluidigm). IMC acquisition stability was monitored by interspersed acquisition of isotope-containing polymer (Fludigm). All successful image acquisitions were processed, and images containing pan-marker staining variation specific to TMA location were removed. In few cases the acquisition was interrupted and later continued resulting in 2 tumor images of the same patient. Therefore, the 281-patient cohort resulted in 289 tumor, 87 healthy breast and 5 liver control images. Where applicable, signal spillover between channels was corrected using functions from the CATALYST R package (version 1.5.6) ${ }^{28}$. During analysis of the samples from University Hospital Zurich, cells from images in one row of the TMA were almost exclusively assigned to the phenotype with the lowest overall marker expression. These samples were poorly stained and had to be excluded from further analysis (images Ay.x1). The 72-patient cohort resulted in 263 tumor, 68 healthy breast and 6 control images used for analysis.

\section{Data processing}

Data were converted to .tiff format and segmented into single cells using the flexible analysis pipeline available at https://github.com/BodenmillerGroup/ImcSegmentationPipeline. Briefly, individual cells and tumor/stroma regions were segmented using a combination of llastik 1.1.9 $9^{29}$ and CellProfiler 2.1.1 $1^{30}$. Ilastik was used to generate a probability map by classifying pixels 
(Single cells - nuclei, membrane and background; Tumor/Stroma - tumor, stroma and background) based on a combination of membrane and nuclei identifying antibody stains. Probability maps were then segmented into single cell, or tumor and stroma object masks using CellProfiler.

Single-cell segmentation masks and tiff images of the 35 channels were overlaid and single-cell marker expression means and spatial features were extracted using the Matlab toolbox regionprops, as implemented in histoCAT ${ }^{31}$. Even with very good quality segmentation, the imaging of tissue segments results in single-cell data of tissue slices and overlapping cell fragments that do not always capture the nucleus of a cell, and therefore nuclei-mismatched signal can be assigned to neighboring cells in densely packed areas. This can lead to rare cases where data assigned to one cell contains marker expression from the neighborhood.

The single cell IDs of each cell's direct neighbors within 4 pixels $(4 \mu \mathrm{m})$ of the cell of interest was detected and recorded using histoCAT software. The number of pixels expanded to detect neighbors was chosen such that small gaps in segmentation would be bridged, yet no cells after the direct neighbor would be recorded (cell minor axis lengths: 5 th - 95th percentile 4.84 14.59 pixels, average 9.51 pixels).

Individual cell locations inside or outside of a tumor mask were identified and the distance of each cell to the tumor boundary (from inside and outside of the tumor region) was calculated using the Matlab toolbox regionprops. Distances were measured between the closest pixels of the objects in question.

\section{Data transformation and normalization}


The presented data were not transformed, and all analyses were based on raw IMC measurements. Single-cell marker expressions are summarized by mean pixel values for each channel. The single-cell data were censored at the 99th percentile to remove outliers, and Zscored cluster means were visualized in heatmaps. For tSNE and PhenoGraph the data were normalized to the 99th percentile, as suggested by the authors of these algorithms ${ }^{32,33}$. To visualize the number of cells per image or patient and for survival modelling, the counts were normalized by the image area (total number of pixels) and displayed as cell density. For coxph survival modelling, these densities were multiplied by a factor of $10^{31}$ in order to yield values larger than 1 and then log-transformed.

\section{Analysis workflow}

The single-cell analysis pipeline was implemented in $\mathrm{R}$, but image analysis steps were performed in Matlab. All statistical tests were performed using common R functions.

\section{Clustering and metaclustering}

Single cells of the large cohort from University Hospital Basel were clustered into groups of phenotypically similar cells using a combination of PhenoGraph ${ }^{33}$ for initial, unsupervised clustering and an aggregation of these clusters into larger groups based on their mean marker correlations to identify cellular metaclusters. In a first step, the data were over-clustered to detect and separate rare cell subpopulations. PhenoGraph (version 2.0) was used with default parameters (as implemented in histoCAT/Cyt) and 20 nearest neighbors. For high-dimensional

clustering, 29 markers and 4 cell shape features were used: Iridium, Histone, phospho Histone, 
CK14, CK5, CK8/18, CK19, CK7, panCK, E/P-Cadherin, ER, PR, HER2, GATA3, SMA, Vimentin, Fibronectin, vWF/CD31, CD44, CD45, CD68, CD3, CD20, cleaved Caspase 3/cleaved PARP, Carbonic Anhydrase, phospho-S6, Ki67, p53, EGFR, Area, Eccentricity, Extent, and Number Neighbors. Of the resulting 71 clusters, the 59 epithelial ones were aggregated into larger groups following the hierarchical clustering (Euclidean distance and Ward's linkage) of their mean marker correlations. Multiscale bootstrap resampling was used to assess the uncertainty of each subtree (R package pvclust, version 2.0), and separation of the hierarchy was assigned so that significant epithelial subtrees were maintained and known biologic differences were separated. This resulted in 14 tumor cell metaclusters of varying size and subtree robustness (Extended Data 5a). Clusters showing marker expression typical of stromal and immune cells, which were limited due to our tumor marker focused panel, were kept as in the original PhenoGraph clustering and not aggregated into larger groups. This metaclustering yielded 27 cellular subgroups, representing various immune, stromal, and epithelial cell types. The granularity, the level and detail at which phenotypes are divided or clustered, of the studied cell types depends on the selection of both the panel and the choice of parameters. While a more granular distinction of cell types might elucidate even more subtle difference in the marker expressions of cells, it would limit comparability between tumors as many tumor cell types would be patient specific.

\section{Cluster matching across cohorts}

Single cells from the second cohort from University Hospital Zurich were clustered unsupervised and independently using PhenoGraph ${ }^{33}$ with the same settings described above for the first cohort and a nearest neighbor parameter of 30 . The clusters were matched to the most 
similar metacluster of the previous cohort using Pearson correlation of the z-scored mean marker expressions. In two special cases (clusters 8 and 15) where the cluster in question was rather poorly correlated with all metaclusters but most correlated with a stromal cell type, we manually re-assigned the cluster because upon visual inspection of the images those clusters represented cells forming clear tumor bulks.

\section{bh-tSNE}

For visualization, high-dimension single-cell data were reduced to two dimensions using the non-linear dimensionality reduction algorithm $\mathrm{tSNE}^{32}$. We applied the Barnes-Hut implementation of tSNE (bh-tSNE) to 99th-percentile normalized data with default parameters (initial dimensions, 110; perplexity, 30; theta, 0.5). The algorithm was run on a randomly subsampled set of cells ( $20 \%$ from each image) in order to not obscure visible patterns in crowded plots and for better computational performance.

\section{Neighborhood analysis}

To identify significantly enriched or depleted pairwise neighbor interactions between cell types, histoCAT functions were used to perform a permutation test based analysis of spatial single-cell neighborhoods ${ }^{31}$. Neighboring cells were defined as those within 4 pixels $(4 \mu \mathrm{m})$. A pvalue cut-off of $<0.01$ was used for significance.

\section{Single-cell pathology patient grouping}


Patients were grouped based on the proportions of tumor cell metaclusters using the cytofkit R implementation of PhenoGraph ${ }^{33}$ (version 1.10.0) with 8 nearest neighbors and default parameters. The parameter number of nearest neighbors was chosen such that small groups of patients consisting of a distinct predominant cell type could be separated. A choice of a higher value for this parameter would lead to fewer groups, and hence patients with entirely unrelated predominant phenotypes grouped together. A lower value of the nearest neighbor parameter might capture more subtle differences in cellular composition of tumor types but would severely limit statistical power for group comparison and survival analysis. The composition of each patient group by their clinically assigned metadata is available in Supplementary Table 3. Patient group 18 was removed from further downstream analysis, due to lack of statistical power, as it contains only three patients with distinct tumors strongly dominated by a rare $\mathrm{HR}^{+} / \mathrm{CK}^{-}$cell type.

\section{Single-cell pathology group matching}

Tumor cores from the second cohort from University Hospital of Zurich were assigned to the most similar previously defined single-cell pathology group based on their matched tumor cell type components. The inverse of Pearson correlation was used as distance metric.

\section{Spatial heterogeneity}

In the cohort from University Hospital Zurich that contains multiple cores per tumor, intra-core heterogeneity of tumor and stromal cells were separately quantified using the entropy based Shannon index on the amounts of the different cell types within each core. Shannon entropy has been shown to serve as a measure of diversity and homogeneity in various 
contexts $^{34}$. It can also be considered a measure for the information content of a string, where in our case every cell of an image is represented by a letter according to its cell type. The most compressible string is obtained if every cell is of the same type, and the string with most information is obtained if every cell is unique (Formula 1, where $\mathrm{P}_{\mathrm{i}}$ is the probability of a given symbol). Inter-core heterogeneity within a tumor was approximated by calculating the KullbackLeibler divergence from the cell-type distribution (proportions of each cell type) of an individual core to the average cell-type distribution across all cores of a patient. Kullback-Leibler divergence describes the information loss when going from an original distribution to a summary distribution ${ }^{35}$. Hence, if all cores of a tumor are composed of identical proportions of the same cell types, the Kullback-Leibler divergences of every individual core to the patient average will be minimal. The R package entropy (version 1.2.1) was used for the calculation of both Shannon entropy and the Kullback-Leibler divergence. Intra-tumor heterogeneity and the consistency of SCP group assignment of images of the same patient are visualized in Extended Data 10.

$$
H(X)=-\sum p_{i} \log _{2}\left(p_{i}\right)
$$

(Formula 1)

\section{Spatial communities}

The images were converted into topological neighborhood graphs where every cell is represented by a node (visualized at the centroid), and the nodes are connected by an edge if the cells directly neighbor each other (Figure 2). Neighboring cells were defined as those within 4 pixels $(4 \mu \mathrm{m})$ of the outermost pixel assigned to a cell. Subsequently, the Louvain community detection algorithm ${ }^{36}$ ( $\mathrm{C}$ implementation by Lefebvre and Guillaume, version 0.2 , wrapped by 
Matlab as used by the implementation of PhenoGraph 2.0 used by histoCAT/Cyt) was applied to identify highly interconnected spatial subunits in the tissue graph. While using community detection algorithms on spatially constrained networks is known to hide underlying non-spatially driven solutions, the only aim of applying the algorithm here was to extract spatial information and identify communities based on physical proximity ${ }^{37}$. This analysis was performed on epithelial cells only to identify tumor communities (without including stromal or immune cells in the graph) and again on all cells of a tissue to identify tumor-microenvironment communities. A tumor-specific cohesiveness score was calculated based on the average sizes of the identified tumor communities. Communities involving fewer than 10 cells were excluded from further analysis in order to focus on cohesive cell patches and not individual disconnected cells. 15 patients were excluded from analysis based on tumor communities because the imaged regions did not contain any tumor communities consisting of at least 10 cells. In order to identify recurring similar spatial cell type communities, the cytofkit PhenoGraph ${ }^{33}$ (version 1.10.0) was run on the min-max normalized, absolute numbers of cells of each cell metacluster in each community. This analysis was conducted separately for the tumor communities based on only the epithelial cell types $(k=80)$ and for the microenvironment communities based on all cells but only taking into account the individual stromal cell types and aggregating all tumor cell types into one label (cell type group 100: including all tumor cells, $\mathrm{k}=30$ ). This analysis was conducted separately for each cohort but based on the matched metacluster cell types.

\section{Stromal environments}


Based on their microenvironment community compositions, images were grouped into 11 different stromal environments using hierarchical clustering (Euclidean distance and Ward's linkage). This analysis was conducted separately for each cohort but based on the matched metacluster cell types.

\section{Overlapping classifications and enrichments}

Fisher's exact test was used to identify single-cell pathology patient groups enriched for a specific stromal environment (Extended Data 8). The test was performed using the R function fisher.test (with parameter enrichment = "greater") for every potential stromal region of a patient group. The p-values were corrected for multiple testing using the Bonferroni method. This enrichment analysis was also conducted with different combinations of single-cell pathology subgroups, stromal environments, and clinical classifications (Extended Data 8).

\section{Survival curves and coxph regression models}

Kaplan-Meier survival curves and coxph survival regression models were generated using the R package survival (version 2.42-4). The overall survival as well as the disease-free survival of patients in different clinical or single-cell-defined subgroups was analyzed (Figure 4, Extended

Data 9, Supplementary Table 4-8). Both logrank tests and coxph models were employed to investigate whether a patient subgroup significantly deviated from the survival of the remaining patients or from the survival of other patients of similar SCP groups or the same clinical classification (Figure 4, Supplementary Table 4-7). Log-transformed densities of communities or single cells, alongside the clinical subgrouping and grading, were provided to a coxph survival 
model in order to find significant associations of certain community or single-cell types with patient risk and to investigate the hazard ratios (Figure 4). Nested coxph models were compared using likelihood ratio tests (anova.coxph) to assess whether additional variables improved the survival model (Supplementary Table 8). 


\section{References:}

25. Kononen, J. et al. Tissue microarrays for high-throughput molecular profiling of tumor specimens. Nat. Med. 4, 844-847 (1998).

26. Kündig, P. et al. Limited utility of tissue micro-arrays in detecting intra-tumoral heterogeneity in stem cell characteristics and tumor progression markers in breast cancer. Journal of Translational Medicine, 16(1), 118 (2018).

27. Giesen, C. et al. Highly multiplexed imaging of tumor tissues with subcellular resolution by mass cytometry. Nat. Methods 11, 417-422 (2014).

28. Chevrier, S. et al. Compensation of Signal Spillover in Suspension and Imaging Mass Cytometry. Cell Syst. 6, 612-620 (2018).

29. Sommer, C., Straehle, C., Köthe, U. \& Hamprecht, F.A. in Proc. 2011 8th IEEE International Symposium on Biomedical Imaging: From Nano to Macro 230-233 (IEEE, 2011).

30. Carpenter A.E., et al. CellProfiler: image analysis software for identifying and quantifying cell phenotypes. Genome Biology 7:R100. PMID: 17076895 (2006) .

31. Schapiro, D. et al. histoCAT: analysis of cell phenotypes and interactions in multiplex image cytometry data. Nat. Methods 14, 873-876 (2017).

32. Amir, A.D. et al. viSNE enables visualization of high dimensional single-cell data and reveals phenotypic heterogeneity of leukemia. Nat. Biotechnol. 31, 545-552 (2013).

33. Levine, J.H. et al. Data-Driven Phenotypic Dissection of AML Reveals Progenitor-like Cells that Correlate with Prognosis. Cell 162, 184-197 (2015).

34. Angel Martín, M., \& Rey, J.-M. (2000). On the role of Shannon's entropy as a measure of heterogeneity. Geoderma (Vol. 98).

35. Kullback, S., Leibler, R. A. On Information and Sufficiency. Ann. Math. Statist. 22 (1951).

36. Blondel, V.D. et al. Fast unfolding of communities in large networks. J. Stat. Mech. (2008) P10008 
37. Expert, P., Evans, T. S., Blondel, V. D., \& Lambiotte, R. Uncovering space-independent communities in spatial networks. Proceedings of the National Academy of Sciences, 108(19), 7663 LP-7668 (2011).

\section{Acknowledgements}

We are grateful for the donation of tumor samples by patients undergoing surgery. We thank Serenella Eppenberger and Susanne Dettwiler for the coordination of tissue collection and for construction of tissue microarrays. We thank the Bodenmiller lab, in particular Natalie de Souza for help with writing the manuscript, and Dr. Daniel Schulz for fruitful discussions. BB's research was funded by a SNSF R`Equip grant, a SNSF Assistant Professorship grant, the SystemsX Transfer Project "Friends and Foes", the SystemX grants Metastasix and PhosphoNEtX, a NIH grant (UC4 DK108132), the CRUK IMAXT Grand Challenge, and by the European Research Council (ERC) under the European Union's Seventh Framework Program (FP/2007-2013)/ERC Grant Agreement n. 336921. HWJ was funded by SystemsX Transitional Post-Doctoral Fellowship, the Canadian Institute of Health Research Post-Doctoral Fellowship, and the Cancer Research Society Scholarship for the Next Generation of Scientists.

\section{Author competing interest}

The authors declare that they have no conflicts of interest. 


\section{Author Information}

These authors contributed equally: Hartland W. Jackson and Jana R. Fischer

\section{Affiliations}

Department of Quantitative Biomedicine, University of Zurich, Switzerland

Hartland W. Jackson, Jana R. Fischer, Vito R.T. Zanotelli, H. Raza Ali, Bernd Bodenmiller

Life Science Zurich Graduate School, ETH Zurich and University of Zurich, Switzerland

Jana R. Fischer, Vito R.T. Zanotelli

CRUK Cambridge Institute, University of Cambridge, Cambridge, UK

H. Raza Ali

Department of Surgery, University of Basel and University Hospital Basel, Switzerland

Robert Mechera, Walter P. Weber

Visceral Surgery Research Laboratory, Clarunis, Department of Biomedicine, University of Basel,

Basel, Switzerland

Savas D. Soysal

Department of Surgery, Clarunis University Center for Gastrointestinal and Liver Diseases Basel,

Basel, Switzerland

Savas D. Soysal

Institute of Pathology and Molecular Pathology, University Hospital Zurich, Zurich, Switzerland

Holger Moch, Zsuzsanna Varga

Institute of Pathology and Genetics, University Hospital Basel, Switzerland

Simone Muenst 


\section{Contributions}

HWJ and BB conceived the study. HWJ performed all image quantification and IMC experiments. ZV performed immunohistochemical staining. JRF performed data analysis. VRTZ constructed image processing and analysis tools. HWJ and JRF performed the biological analysis and interpretation with input from the co-authors. RA provided input on clinical interpretation and survival analysis. HM, ZV, RM, SDS, SMS, and WPW provided patient samples and clinical input throughout the study. HWJ, JRF, and BB wrote the manuscript.

\section{Corresponding Author}

Bernd Bodenmiller (bernd.bodenmiller@uzh.ch)

\section{Data availability}

The data supporting the findings of this study, including high-dimension tiff images, single-cell and tumor/stroma masks, single-cell and patient data will be available online upon publication (DOI: 10.5281/zenodo.3518284).

\section{Code availability}

All code that produced the results of this study will be available on https://github.com/BodenmillerGroup/SCPathology publication upon publication.

\section{Extended data legends}

Extended Data 1: Antibody panel and example 6-marker pseudo-colored images. 
Antigens targeted by the antibodies in the panel of 35 isotope-conjugated antibodies used to stain the breast cancer tissue. Representative marker images from the analysed cohort generated by IMC. Every marker is visualized at least once. Each image represents a different tumor of the analysed cohort. Each marker was individually scaled to enable visualization. Scale bar $=100 \mu \mathrm{m}$.

Extended Data 2: IHC and IMC comparison and reproducibility analysis.

(a) Representative IMC and immunohistochemistry (IHC) images of the quantified stains in sections of the same tumor core. (b) Scatter plot and correlation of total IHC and IMC signal in sections of the same tumor core (IHC = optical density $/ \mu \mathrm{m}^{2}, \mathrm{IMC}=$ ion counts $/ \mu \mathrm{m}^{2}, \mathrm{n}=319$ cores). (c) Scatter plot and correlation of the number of positively stained cells in sections from the same tumor core ( $n=319$ cores). (d) Bland-Altman plots for reproducibility of IMC signal in positively stained cells across images from different regions of the same tumor adapted to visualize the average across four samples on the $\mathrm{x}$-axis and the difference of every individual sample to the tumor average on the $y$-axis. Only images containing positively stained cells and more than 200 cells in total were taken into account for this analysis (ER: $n=280$ cores from 72, PR: $n=213$ cores from 66 patients, HER2: $n=291$ cores from 72 patients, Ki67: $n=281$ cores from 72 patients, E/P-Cadherin: $n=200$ cores from 65 patients). The red line represents the overall average of the differences to the tumor mean, and the blues lines represent the $95 \%$ confidence interval (1.96*standard deviation). The percentage of observations that fall within the confidence interval is indicated at the top of each plot.

Extended Data 3: Simultaneous IF and IMC imaging. 
Immunofluorescence (IF) and Mass Cytometry (IMC) imaging of the same tissue sample using metal-conjugated HER2 and pan-Cytokeratin primary antibodies and both fluorescent and metalconjugated secondary stains. Pseudo-color images of (a) individual channels, (b) three marker images produced from each label type (White: overlap, Red: HER2, Green: pan-Cytokeratin, Blue: DNA Intercalator), as well as (c) an overlay of the same marker from all three label types (White: overlap, Red: Secondary IF, Green: Secondary IMC, Blue: Primary IMC). (d) High magnification image of regions labelled by white squares in panel $b$ comparing immunofluorescence and imaging mass cytometry resolution.

Extended Data 4: Single-cell localization relative to tumor-stroma interface. tSNE maps of 171,288 subsampled single cells from high-dimension images of breast tumors colored by (a) patient, (b) localization relative to the tumor-stroma interface, (c) single-cell distance to the tumor-stroma interface and (d) number of neighboring cells. (e-g) Representative image with single-cell mask labeled by (e) metacluster identifier , (f) tumor and stroma masks, and $(\mathbf{g})$ heatmap representing distances of single cells to the tumor-stroma interface from each side. Scale bar $=100 \mu \mathrm{m} .(\mathbf{h}-\mathbf{j})$ Log-transformed distances to tumor front of (h) stromal-cell clusters and (i) tumor-cell metaclusters, and (j) binned distances of all metaclusters to the tumor front, where bin number 0 contains all cells that are directly touching the interface. Negative distances represent the distance to the tumor boundary from inside the tumor and positive values indicate distance outside the tumor.

Extended Data 5: Metaclustering and cluster matching across cohorts. 
(a) Heatmap displaying z-scored mean marker expressions of single-cell phenotypic clusters identified by PhenoGraph (Figure 1) with colors on colorbar and hierarchical clustering indicating the corresponding metacluster. Red stars on the hierarchical clustering tree indicate subgroups that robustly reappear as separate groups using multiscale bootstrap resampling ( $R$ function pvclust, $p<0.05$ ). (b) Examples of untransformed distributions of cluster marker expressions differing between metaclusters. (c) Heatmaps showing the z-scored mean marker expression or distance to tumor-stroma interface for each metacluster defined in the Basel 281-patient cohort and each matched PhenoGraph cluster from the Zurich multi-core 71-patient cohort. PhenoGraph clusters of the Zurich cohort were matched to the metaclusters of the Basel cohort based on Pearson correlation of the mean marker expression (Methods).

Extended Data 6: Densities of single-cell phenotypes in different clinical subtypes and SCP patient subgroups.

Box plots of cellular metacluster densities in patients of (a) each clinical subtype (HR+HER2-: $n=$ 173, HR+HER2+: $n=29$, HR-HER2+: $n=23$, TripleNeg: $n=48$ ) and (b) each SCP subgroup (center line, median; box limits, first and third quartile; whiskers, 1.5x interquartile range; points beyond whiskers, outliers; SCP1: $n=17$, SCP2: $n=21$, SCP3: $n=20$, SCP4: $n=12$, SCP5: $n=32$, SCP6: $n=$ 10, SCP7: $n=13$, SCP8: $n=11$, SCP9: $n=20$, SCP10: $n=24$, SCP11: $n=31$, SCP12: $n=14$, SCP13: $n=15$, SCP14: $n=11$, SCP15: $n=8$, SCP16: $n=10$, SCP17: $n=9$, SCP18: $n=3$ ).

Extended Data 7: Stromal environments based on microenvironment community composition and their distinct pairwise cell type interactions. 
(a) Hierarchical clustering of tumor cores $(n=281)$ according to stromal community content and splitting into corresponding stromal environments $(n=11)$. The stacked bar plot at the top indicates the average number of cells from each cellular metacluster present within each microenvironment community type. (b) The presence of significant $(p<0.01)$ cell-cell interactions (red) and cell-cell avoidances (blue) identified per image based on a permutation test (1000 permutations). Black outlined regions indicate significant interactions that are enriched in images from the respective stromal environments (one-sided Fisher's exact test for enrichment, $p<0.05$ after multiple-testing correction). Color bars on the right indicate the SCP subgroup, grade, and clinical subtype of the tumor. Cell-type interactions along the top are indicated by the labeled cell type of interest and neighboring cell.

Extended Data 8: Comparisons and enrichments between classifications.

Bubble plot visualizing overlap between SCP subgroups of breast cancer (SCP1: $n=17, \operatorname{SCP} 2: n=$ 21, SCP3: $n=20$, SCP4: $n=12$, SCP5: $n=32$, SCP6: $n=10$, SCP7: $n=13$, SCP8: $n=11$, SCP9: $n=$ 20, SCP10: $n=24, \operatorname{SCP} 11: n=31, \operatorname{SCP} 12: n=14, \operatorname{SCP} 13: n=15, \operatorname{SCP} 14: n=11, \operatorname{SCP} 15: n=8, \operatorname{SCP} 16:$ $n=10, \operatorname{SCP} 17: n=9, \operatorname{SCP} 18: n=3$ excluded) and (a) clinical subtypes (HR+HER2-: $n=173$, HR+HER2+: $n=29$, HR-HER2+: $n=23$, TripleNeg: $n=48$ ) and (b) stromal environments (SE1: $n=$ 49, SE2: $\mathrm{n}=88$, SE3: $\mathrm{n}=9$, SE4: $\mathrm{n}=24$, SE5: $\mathrm{n}=25$, SE6: $\mathrm{n}=24$, SE7: $\mathrm{n}=8$, SE8: $\mathrm{n}=14$, SE9: $\mathrm{n}=$ 14, SE10: $n=18$, SE11: $n=2$ ). One-sided Fisher's exact test for enrichment. $\bullet p<0.1,{ }^{*} p<0.05, * *$ $p<0.01,{ }^{* * *} p<0.001$. Exact $p$-values for highlighted pairs: SE1 and SCP7: $p=0.013$, SE3 and SCP8: $p=0.021$, SE5 and SCP15: $p=0.031$ SE6 and SCP3: $p=0.034$, SE8 and SCP14: $p=0.008$, SE8 and SCP8: $p=0.093$, SE9 and SCP12: $p=0.036$, HR+HER2- and SCP3: $p=0.079$, HR+HER2- and SCP5: 
$p=3.58 e-04, H R+H E R 2+$ and SCP2: $p=0.032$, HR-HER2+ and SCP11: $p=2.36 e-04, H R-H E R 2-$ and SCP8: $p=0.060$, HR-HER2- and SCP14: $p=0.008$, HR-HER2- and SCP15: $p=6.13 e-06$, HR-HER2and SCP16: $p=0.031$.

Extended Data 9: Kaplan-Meier survival curves for overall and disease free survival.

Kaplan-Meier survival curves of (a-b) overall survival for Stromal Environments not shown in Figure 4 and (c-I) disease-free survival for each patient group based on (c) clinical subtype , (d) grade, (e-h) SCP subgroup, and (i-I) stromal environment . Two-sided logrank test $\mathbf{p}<0.05$ compared to all other samples (for exact p-values see SI Tables 5 and 7).

Extended Data 10: Multi-core cohort regional heterogeneity analysis.

Quantification of intra-tumor regional heterogeneity in Zurich multi-core cohort. (a) Hierarchically clustered stacked bar plot of cell type metacluster densities in each tumor, grouped by patient. Colored columns indicate patient, clinical subtypes, SCP subgroups, location of core in the tumor, Shannon entropy (intra-core heterogeneity), and tumor-specific cohesiveness score. (b) Dot plot of Kullback-Leibler divergence from the cell-type distribution of an individual tumor region to the patient average distribution, colored according to the SCP subgroup classification per tumor region ( $n=263$ tumor cores), grouped by patient ( $n=71$ patients) and ordered by the increasing average Kullback-Leibler divergence per patient. (c) Boxplot of the same Kullback-Leibler divergences of each region to the patient's average cell-type distribution, grouped by tumor regions individually identified as the same SCP subgroup, independent of patient (center line, median; box limits, first and third quartile; whiskers, $1.5 x$ interquartile range; 
points beyond whiskers, outliers; SCP1: $n=12$, SCP2: $n=13$, SCP3: $n=11$, SCP4: $n=10$, SCP6: $n$ = 76, SCP7: $n=7$, SCP8: $n=3$, SCP9: $n=5$, SCP10: $n=1$, SCP11: $n=26$, SCP12: $n=51$, SCP13: $n=$ 15, SCP14: $n=18$, SCP15: $n=4$, SCP16: $n=5$, SCP17: $n=5$, SCP18: $n=1)$. (d) Bar indicating the percentage of patients $(n=71)$ with the indicated fraction of individually classified images matching the whole tumor classification. (e) Bubble plot visualizing intra-tumor region variation within patients of each SCP subgroup. Rows represent tumors of each SCP subgroup as identified by the combined analysis of all imaged regions. Columns represent tumor regions individually matched to a SCP subgroup. For each whole-tumor classification on the $y$-axis, circle size indicates the fraction of corresponding images individually classified as a SCP subgroup. For each image classification on the $\mathrm{x}$-axis, color indicates the fraction of images within each tumor type. 